\title{
HYPERBOLIC GEOMETRY AND HÖLDER CONTINUITY OF CONFORMAL MAPPINGS
}

\author{
RAIMO NÄKKI ${ }^{(*)}$ and BRUCE PALKA
}

\section{Introduction}

Let $A$ be a set in the complex plane $C$ and let $0<\alpha \leqq 1$. A complex-valued function $f$ defined on $A$ is a member of the class $\operatorname{Lip}_{\alpha}(A)$, the Lipschitz class in $A$ with exponent $\alpha$, if $f$ satisfies a uniform Hölder condition with exponent $\alpha$ :

$$
|f(z)-f(w)| \leqq M|z-w|^{\alpha}
$$

for all $z$ and $w$ in $A$, where $M$ is a positive constant.

Consider now a simply connected proper subdomain $D$ of $\boldsymbol{C}$ and a conformal mapping $f$ of $D$ onto the unit disk $B=\{z:|z|<1\}$. In general, it cannot be inferred either that $f$ belongs to $\operatorname{Lip}_{\alpha}(D)$ for some $\alpha$ or that $f^{-1}$ belongs to $\operatorname{Lip}_{\beta}(B)$ for some $\beta$, even if $f$ is known to extend to a homeomorphism between $\bar{D}$ and $\bar{B}$. Whether one or both of these inferences can be drawn depends in a critical way on the geometry of $D$. This principle is certainly well understood and a search of the literature reveals a number of geometric criteria which, when satisfied by $D$, are sufficient to insure either that $f$ or that $f^{-1}$ belongs to some Lipschitz class. (See, for instance, [8], [9], [10], [11] or [14].) While these criteria are more than adequate for the purposes of most applications, they are unsatisfying in one significant regard: they fail to actually characterize the domains $D$ for which $f$ is uniformly Hölder continuous or those domains for which $f^{-1}$ enjoys this property. The reason for this appears to be that the criteria under discussion are too closely bound to the euclidean geometry of $D$. The precise relationship between the euclidean geometry of $D$ and uniform Hölder continuity on the part of $f$ or $f^{-1}$ is, to judge by available evidence, a subtle one indeed.

In a recent paper [1] Becker and Pommerenke elected to abandon the euclidean perspective and to examine Lipschitz classes from the point of view of hyperbolic geometry. They demonstrated that a conformal mapping $f$ of the unit disk $B$ onto a domain $D$ will belong to $\operatorname{Lip}_{\beta}(B)$ if and only if, corresponding to some (each)

(*) Part of this research was done while the author was visiting The University of Texas at Austin in 1983-84. 
point $z_{0}$ in $D$, there exists a constant $b>0$ such that

$$
h_{D}\left(z_{0}, z\right) \leqq \frac{1}{\beta} \log \frac{b}{d(z, \partial D)}
$$

for all $z$ in $D$. Here $h_{D}$ designates the hyperbolic distance in $D$ and $d(z, \partial D)$ denotes the euclidean distance from $z$ to $\partial D$.

It is natural to seek an analogue to the above proposition for mappings onto the unit disk, that is, to attempt to characterize in terms of hyperbolic geometry the domains $D$ with the property that a conformal mapping $f$ of $D$ onto $B$ belongs to $\operatorname{Lip}_{\alpha}(D)$. In the present paper we obtain such a characterization. It should be emphasized at the very outset that the presence of $f$ in $\operatorname{Lip}_{\alpha}(D)$ is compatible with an appreciably greater degree of topological irregularity in $\partial D$ than can arise in the context of Becker's and Pommerenke's result. This added complexity partly accounts for the fact that we formulate our characterization in terms of the geodesic cross-cuts of $D$ and not in terms of an estimate akin to (1). In fact, it is not generally true that the obvious analogue to (1) in the current setting, an inequality of the form

$$
h_{D}\left(z_{0}, z\right) \geqq \alpha \log \frac{a}{d(z, \partial D)},
$$

is sufficient to place $f$ in $\operatorname{Lip}_{\alpha}(D)$, although with appropriate restrictions on $D$ we are able to salvage a facsimile of the Becker-Pommerenke theorem involving (2). The consideration of geodesic cross-cuts leads, in addition, to an alternative to (1) as a means of characterizing the domains $D$ such that a conformal mapping of $B$ onto $D$ belongs to $\operatorname{Lip}_{\beta}(B)$. Finally, we indicate several characterizations of the domains which are the images of $B$ under conformal quasi-isometries.

\section{Hyperbolic geometry}

The Poincaré density $\lambda_{D}$ is defined for any simply connected proper subdomain $D$ of $C$ by the equation

$$
\lambda_{D}(z)=\frac{2\left|f^{\prime}(z)\right|}{1-|f(z)|^{2}},
$$

where $f$ is a conformal mapping of $D$ onto $B$. As is well known, this definition is independent of the particular choice of $f$. Associated to the density $\lambda_{D}$ is a conformally invariant distance $h_{D}$ in $D$, defined by

$$
h_{D}(z, w)=\inf _{\gamma} \int_{\gamma} \lambda_{D}(\zeta)|d \zeta|,
$$

with the infimum extended over the family of rectifiable paths $\gamma$ joining $z$ and $w$ in $D$. The distance $h_{D}$ is often referred to as the hyperbolic distance in $D$, for the reason that, when equipped with this distance, the domain $D$ provides a model for plane 
hyperbolic non-euclidean geometry. The lines in this model are the trajectories of the geodesics for the Poincaré metric in $D$, the Riemannian metric corresponding to the fundamental form $d s=\lambda_{D}(z)|d z|$. These hyperbolic lines will henceforth be referred to simply as the geodesics of $D$. Each such geodesic is an open arc, i.e., is homeomorphic to the real line. Of course, in the case $D=B$ one obtains the familiar Poincaré model for plane hyperbolic geometry, in which the geodesics are the intersections of $B$ with euclidean lines through the origin or with euclidean circles orthogonal to $\partial B$. We recall the formula

$$
h_{B}(0, z)=\log \frac{1+|z|}{1-|z|},
$$

which has the consequence that the geodesic $\gamma$ of $B$ terminating in the points $z$ and $w$ on the unit circle satisfies

$$
\log \frac{2}{|z-w|} \leqq h_{B}(0, \gamma) \leqq \log \frac{4}{|z-w|} .
$$

(The notation $h_{D}(z, A)$ will indicate the hyperbolic distance from a point $z$ in a domain $D$ to a subset $A$ of $D$.)

Of special significance in this paper will be the geodesics of a domain $D$ which are also cross-cuts of $D$. We draw attention to the fact that not every geodesic of $D$ need have this property. On the other hand, two classical results associated with the theory of prime ends insure that each pair of accessible boundary points of $D$ is the set of terminal points of at least one - and possibly more than one - geodesic cross-cut of $D$. To be specific, let $f$ map $D$ conformally onto $B$ and let $E$ be an end-cut of $D$ terminating at a point $z$ of $\partial D$. A theorem of Koebe states that $f$ possesses a limit $z^{\prime}$ at $z$ along $E$, while a theorem of Lindelöf then asserts that the mapping $f^{-1}$ must have $z$ as its non-tangential limit at $z^{\prime}$. In particular, $f^{-1}$ has $z$ as its limit at $z^{\prime}$ along any geodesic of $B$ terminating at $z^{\prime}$. A good reference for these and related results is [2], especially Chapter 9.

Before proceeding we alert the reader to one notational convention observed throughout this article: the notations $\bar{D}$ and $\partial D$ are used to designate the closure and boundary of a domain $D$ relative to $C$, not relative to the extended complex plane.

\section{Lipschitz classes and geodesic cross-cuts}

Let $f$ map a plane domain $D$ conformally onto $B$. In this section we characterize in terms of geodesic cross-cuts those domains $D$ for which $f$ belongs to $\operatorname{Lip}_{\alpha}(D)$ and those for which $f^{-1}$ is a member of $\operatorname{Lip}_{\beta}(B)$.

Theorem 1. Let $f$ be a conformal mapping of a domain $D$ onto $B$ and let $0<<\leqq 1$. Then $f$ belongs to $\operatorname{Lip}_{\alpha}(D)$ if and only if, corresponding to some (each) 
point $z_{0}$ in $D$, there exists a constant $a>0$ such that

$$
h_{D}\left(z_{0}, \gamma\right) \geqq \alpha \log \frac{a}{|z-w|},
$$

whenever $z$ and $w$ are distinct points on $\partial D$ and $\gamma$ is a geodesic cross-cut of $D$ terminating in $z$ and $w$.

Proof. For the necessity, fix a point $z_{0}$ in $D$. We may assume that $f\left(z_{0}\right)=0$. We may further assume that $f$ is defined and continuous on $\bar{D}$ and that $f$ belongs to $\operatorname{Lip}_{\alpha}(\bar{D})$. Let $M>0$ be a Lipschitz constant for $f$ corresponding to the exponent $\alpha$. If $\gamma$ is a geodesic cross-cut of $D$ terminating in the distinct points $z$ and $w$ on $\partial D$, then in view of (3)

$$
h_{D}\left(z_{0}, \gamma\right)=h_{B}(0, f(\gamma)) \geqq \log \frac{2}{|f(z)-f(w)|} \geqq \alpha \log \frac{a}{|z-w|},
$$

where $a=(2 / M)^{1 / \alpha}$. This establishes (4).

In proving the converse assertion, we fix a point $z_{0}$ in $D$ for which (4) is valid and again assume, as we may, that $f\left(z_{0}\right)=0$. We begin by demonstrating that $f$ can be extended to a continuous mapping of $\bar{D}$.

Consider a point $z$ of $\partial D$ and suppose that $f$ fails to have a limit at $z$. Then we can choose sequences $\left\langle z_{k}\right\rangle$ and $\left\langle w_{k}\right\rangle$ in $D$ such that $z_{k} \rightarrow z$ and $w_{k} \rightarrow z$, while $f\left(z_{k}\right) \rightarrow z^{\prime}$ and $f\left(w_{k}\right) \rightarrow w^{\prime}$, where $z^{\prime} \neq w^{\prime}$. An elementary geometric argument establishes the existence of end-cuts $E_{k}$ and $F_{k}$ of $D$ joining $z_{k}$ and $w_{k}$, respectively, to distinct points $z_{k}^{*}$ and $w_{k}^{*}$ on $\partial D$ and satisfying $\operatorname{dia}\left(E_{k}\right) \rightarrow 0$ and $\operatorname{dia}\left(F_{k}\right) \rightarrow 0$, as $k \rightarrow \infty$. Standard extremal length considerations reveal that $\operatorname{dia}\left[f\left(E_{k}\right)\right] \rightarrow 0$ and dia $\left[f\left(F_{k}\right)\right] \rightarrow 0$. Furthermore, classical theorems of Koebe and Lindelöf assert that $f\left(E_{k}\right)$ and $f\left(F_{k}\right)$ are end-cuts of $B$ terminating at certain points $z_{k}^{\prime}$ and $w_{k}^{\prime}$, respectively, on the unit circle and that the mapping $f^{-1}$ has non-tangential limits $z_{k}^{*}$ at $z_{k}^{\prime}$ and $w_{k}^{*}$ at $w_{k}^{\prime}$. Let $\gamma_{k}^{\prime}$ be the geodesic of $B$ with terminal points $z_{k}^{\prime}$ and $w_{k}^{\prime}$. Then $\gamma_{k}=f^{-1}\left(\gamma_{k}^{\prime}\right)$ is a geodesic cross-cut of $D$ terminating in $z_{k}^{*}$ and $w_{k}^{*}$. By virtue of (4)

$$
h_{D}\left(z_{0}, \gamma_{k}\right) \geqq \alpha \log \frac{a}{\left|z_{k}^{*}-w_{k}^{*}\right|} \rightarrow \infty,
$$

as $k \rightarrow \infty$, since $z_{k}^{*} \rightarrow z$ and $w_{k}^{*} \rightarrow z$. On the other hand,

$$
h_{B}\left(0, \gamma_{k}^{\prime}\right) \leqq \log \frac{4}{\left|z_{k}^{\prime}-w_{k}^{\prime}\right|} \rightarrow \log \frac{4}{\left|z^{\prime}-w^{\prime}\right|}<\infty,
$$

in light of (3) and the fact that $z_{k}^{\prime} \rightarrow z^{\prime}$ and $w_{k}^{\prime} \rightarrow w^{\prime}$. This contradiction to the conformal invariance of the hyperbolic distance shows that $f$ must have a limit at $z$, an arbitrary point of $\partial D$. We conclude that $f$ admits an extension to a continuous mapping of $\bar{D}$. The notation $f$ will be retained for the extended mapping. 
Let $z$ and $w$ be distinct accessible points on $\partial D$. There is a geodesic cross-cut $\gamma$ of $D$ with $z$ and $w$ as its terminal points. Invoking (3) and (4), we obtain

$$
\alpha \log \frac{a}{|z-w|} \leqq h_{D}\left(z_{0}, \gamma\right)=h_{B}(0, f(\gamma)) \leqq \log \frac{4}{|f(z)-f(w)|},
$$

from which we infer that

$$
|f(z)-f(w)| \leqq M|z-w|^{\alpha},
$$

with $M=4 / a^{\alpha}$. Because the set of accessible boundary points of $D$ is dense in $\partial D$, the continuity of $f$ on $\bar{D}$ implies the validity of this estimate for all points $z$ and $w$ of $\partial D$. Theorem 1 in [4] - see also [13] - insures that $f$ belongs to $\operatorname{Lip}_{\alpha}(D)$.

The analogue to the preceding theorem for mappings from $B$ into the complex plane is:

Theorem 2. Let $f$ be a conformal mapping of $B$ onto a domain $D$ and let $0<\beta \leqq 1$. Then $f$ belongs to $\operatorname{Lip}_{\beta}(B)$ if and only if, corresponding to some (each) point $z_{0}$ in $D$, there exists a constant $b>0$ such that

$$
h_{D}\left(z_{0}, \gamma\right) \leqq \frac{1}{\beta} \log \frac{b}{|z-w|},
$$

whenever $z$ and $w$ are distinct points on $\partial D$ and $\gamma$ is a geodesic cross-cut of $D$ terminating in $z$ and $w$.

Proof. To establish the necessity of condition (5), fix a point $z_{0}$ in $D$. We are here at liberty to assume that $f(0)=z_{0}$. Let $M>0$ be a Lipschitz constant for $f$ corresponding to the exponent $\beta$. If $\gamma$ is a geodesic cross-cut of $D$ terminating in distinct points $z$ and $w$, designate by $z^{*}$ and $w^{*}$ the corresponding terminal points of the geodesic $f^{-1}(\gamma)$ of $B$. We use (3) to infer that

where $b=4^{\beta} M$.

$$
h_{D}\left(z_{0}, \gamma\right)=h_{B}\left(0, f^{-1}(\gamma)\right) \leqq \log \frac{4}{\left|z^{*}-w^{*}\right|} \leqq \frac{1}{\beta} \log \frac{b}{|z-w|},
$$

For the sufficiency, let $z_{0}$ be a point of $D$ for which (5) is satisfied. As earlier, we make the assumption that $f(0)=z_{0}$. We first show that $f$ can be extended to a continuous mapping of $\bar{B}$. To do so, we observe that inequality (5) implies that $|z-w| \leqq b$ for each pair of accessible points $z$ and $w$ on $\partial D$ and, hence, that $D$ is a bounded domain. Fix a point $z$ on $\partial B$. An often cited result of Wolff [15] permits us to select a sequence $\left\langle\alpha_{k}\right\rangle$ of geodesics of $B$ such that $\alpha_{k}$ separates $z$ from 0 and such that both $\operatorname{dia}\left(\alpha_{k}\right) \rightarrow 0$ and $\operatorname{dia}\left(\alpha_{k}^{\prime}\right) \rightarrow 0$, as $k \rightarrow \infty$. Here $\alpha_{k}^{\prime}=f\left(\alpha_{k}\right)$. Let $D_{k}$ designate the component of $B \backslash \alpha_{k}$ not containing the origin and write $D_{k}^{\prime}=f\left(D_{k}\right)$. We claim that $\operatorname{dia}\left(D_{k}^{\prime}\right) \rightarrow 0$, as $k \rightarrow \infty$.

Suppose this not to be the case. Passing to a subsequence, we may then assume that dia $\left(D_{k}^{\prime}\right) \geqq 3 \delta$ for some $\delta>0$ and for all $k$. We may further suppose that $\operatorname{dia}\left(\bar{\alpha}_{k}^{\prime}\right)<\delta$ for all $k$. It is then apparent that $\operatorname{dia}\left(\partial D_{k}^{\prime} \backslash \bar{\alpha}_{k}^{\prime}\right) \geqq 2 \delta$ for all $k$. Conse- 
quently, we can choose for each $k$ a pair of points $z_{k}^{\prime}$ and $w_{k}^{\prime}$ in $\partial D_{k}^{\prime} \backslash \bar{\alpha}_{k}^{\prime}$ which are accessible from $D_{k}^{\prime}$ and which satisfy $\left|z_{k}^{\prime}-w_{k}^{\prime}\right| \geqq \delta$. The classical theorems of Koebe and Lindelöf referred to earlier imply that $z_{k}^{\prime}$ and $w_{k}^{\prime}$ are the non-tangential limits of $f$ at certain points $z_{k}$ and $w_{k}$, respectively, of $\partial D_{k} \backslash \bar{\alpha}_{k}$. Let $\gamma_{k}$ be the geodesic of $B$ with terminal points $z_{k}$ and $w_{k}$. It is clear that $\gamma_{k}$ lies in $D_{k}$. Since dia $\left(D_{k}\right) \rightarrow 0$ by construction, it follows that

$$
h_{B}\left(0, \gamma_{k}\right) \rightarrow \infty
$$

as $k \rightarrow \infty$. On the other hand, $\gamma_{k}^{\prime}=f\left(\gamma_{k}\right)$ is a geodesic cross-cut of $D$ with terminal points $z_{k}^{\prime}$ and $w_{k}^{\prime}$. Referring to (5), we conclude that

$$
h_{D}\left(z_{0}, \gamma_{k}^{\prime}\right) \leqq \frac{1}{\beta} \log \frac{b}{\left|z_{k}^{\prime}-w_{k}^{\prime}\right|} \leqq \frac{1}{\beta} \log \frac{b}{\delta}
$$

for all $k$, which contradicts the conformal invariance of the hyperbolic distance. Thus dia $\left(D_{k}^{\prime}\right) \rightarrow 0$, as asserted. This fact implies that $f$ possesses a limit at $z$, an arbitrary point of $\partial B$. We infer that $f$ admits an extension to a continuous mapping of $\bar{B}$, which extension we continue to denote by $f$.

Now consider points $z$ and $w$ on $\partial B$ such that $f(z) \neq f(w)$. If $\gamma$ is the geodesic of $B$ with endpoints $z$ and $w$, then $f(\gamma)$ is a geodesic cross-cut of $D$ terminating in $f(z)$ and $f(w)$. Applying (3) and (5), we obtain

$$
\log \frac{2}{|z-w|} \leqq h_{B}(0, \gamma)=h_{D}\left(z_{0}, f(\gamma)\right) \leqq \frac{1}{\beta} \log \frac{b}{|f(z)-f(w)|}
$$

which leads to the inequality

$$
|f(z)-f(w)| \leqq M|z-w|^{\beta}
$$

with $M=b / 2^{\beta}$. Since this estimate holds for all $z$ and $w$ on $\partial B$, we can appeal to a well known theorem of Hardy and Littlewood and conclude that $f$ belongs to $\operatorname{Lip}_{\beta}(B)$.

Corollary 1. Let $f$ be a conformal mapping of a domain $D$ onto $B$ and let $0<\alpha, \beta \leqq 1$. Then $f$ belongs to $\operatorname{Lip}_{\alpha}(D)$ and $f^{-1}$ belongs to $\operatorname{Lip}_{\beta}(B)$ if and only if, corresponding to some (each) point $z_{0}$ in $D$, there exist constants $a>0$ and $b>0$ such that

$$
\alpha \log \frac{a}{|z-w|} \leqq h_{D}\left(z_{0}, \gamma\right) \leqq \frac{1}{\beta} \log \frac{b}{|z-w|}
$$

whenever $z$ and $w$ are distinct points on $\partial D$ and $\gamma$ is a geodesic cross-cut of $D$ terminating in $z$ and $w$. 


\section{Lipschitz classes and lower bounds for hyperbolic distance}

Let $f$ map a domain $D$ conformally onto $B$. While Theorem 1 characterizes those $D$ for which $f$ belongs to $\operatorname{Lip}_{\alpha}(D)$, it would be desirable, if for none other than aesthetic reasons, to have a second characterization that more closely mimics the theorem of Becker and Pommerenke cited in the introduction. The presence of $f$ in $\operatorname{Lip}_{\alpha}(D)$ does, in fact, impose a necessary condition analogous to (1) on the domain $D$ : corresponding to some (each) point $z_{0}$ in $D$, there exists a constant $a>0$ such that

$$
h_{D}\left(z_{0}, z\right) \geqq \alpha \log \frac{a}{d(z, \partial D)}
$$

for all $z$ in $D$. Indeed, if we fix $z_{0}$ in $D$, if we assume that $f\left(z_{0}\right)=0$ and if we choose a Lipschitz constant $M>0$ for $f$ corresponding to $\alpha$, we obtain for $z$ in $D$

$$
h_{D}\left(z_{0}, z\right)=h_{B}(0, f(z)) \geqq \log \frac{1}{d(f(z), \partial B)} \geqq \log \frac{1}{M d(z, \partial D)^{\alpha}}=\alpha \log \frac{a}{d(z, \partial D)},
$$

where $a=M^{-1 / \alpha}$. If, however, one looks to condition (6) as a possible sufficient condition for $f$ to be in $\operatorname{Lip}_{\alpha}(D)$, one is destined to be disappointed, for reasons which will now be indicated.

In the first place, the fundamental inequality

$$
\lambda_{D}(z) \geqq \frac{1}{2 d(z, \partial D)},
$$

valid in an arbitrary simply connected proper subdomain $D$ of $C$, has as a consequence that

$$
h_{D}\left(z_{0}, z\right) \geqq \frac{1}{2} \log \frac{d\left(z_{0}, \partial D\right)}{d(z, \partial D)}
$$

for all points $z_{0}$ and $z$ in such a domain. (See Lemma 2.1 in [6].) This means that one could hope to derive information about the Hölder continuity of the mapping $f$ solely on the basis of an estimate such as (6) only when $1 / 2<\alpha \leqq 1$. However, even this restriction on $\alpha$ does not render the situation more promising. A simple example serves to illustrate this point.

Example 1. The domain $D=\left\{e^{\pi z}: z \in B\right\}$ has the property that, corresponding to each point $z_{0}$ in $D$, there exist constants $a>0$ and $b>0$ such that

$$
\log \frac{a}{d(z, \partial D)} \leqq h_{D}\left(z_{0}, z\right) \leqq \log \frac{b}{d(z, \partial D)}
$$

for all $z$ in $D$. The function $f(z)=\pi^{-1} \log z$ maps $D$ conformally onto $B$, but does not belong to $\operatorname{Lip}_{\alpha}(D)$ for any $\alpha>0$. 
The verification of (8) is a straightforward application of the Koebe distortion theorem, once it is observed that $e^{-\pi} / \pi \leqq\left|f^{\prime}(z)\right| \leqq e^{\pi} / \pi$ for all $z$ in $D$. Since $f$ fails to admit a continuous extension to $\bar{D}$, it is evident that $f$ cannot be uniformly Hölder continuous in $D$.

The upshot of the foregoing discussion is that something above and beyond estimate (6) must be known about a domain $D$ if it is to be asseried that a conformal mapping of $D$ onto $B$ belongs to the class $\operatorname{Lip}_{\alpha}(D)$, even in the non-trivial case $\alpha>1 / 2$. The following theorem imposes on $D$ an additional constraint, which enables us to make such an assertion.

Theorem 3. Suppose $D$ has the property that for some constant $c>0$

$$
\max _{\zeta \in \gamma} d(\zeta, \partial D) \leqq c|z-w|,
$$

whenever $z$ and $w$ are distinct points on $\partial D$ and $\gamma$ is a geodesic cross-cut of $D$ terminating in $z$ and $w$. Let $f$ be a conformal mapping of $D$ onto $B$ and let $1 / 2 \leqq$ $\alpha \leqq 1$. Then $f$ belongs to $\operatorname{Lip}_{\alpha}(D)$ if and only if, corresponding to some (each) point $z_{0}$ in $D$, there exists a constant $a>0$ such that

for all $z$ in $D$.

$$
h_{D}\left(z_{0}, z\right) \geqq \alpha \log \frac{a}{d(z, \partial D)}
$$

Proof. The necessity of the estimate (10) for $f$ to be in the class $\operatorname{Lip}_{\alpha}(D)$ was observed earlier. For the sufficiency, fix a point $z_{0}$ in $D$ for which (10) is valid. Let $\gamma$ be a geodesic cross-cut of $D$ with distinct terminal points $z$ and $w$ and let $\zeta_{0}$ be the point on $\gamma$ at minimal hyperbolic distance from $z_{0}$. Then by (10) and (9)

$$
h_{D}\left(z_{0}, \gamma\right)=h_{D}\left(z_{0}, \zeta_{0}\right) \geqq \alpha \log \frac{a}{d\left(\zeta_{0}, \partial D\right)} \geqq \alpha \log \frac{a^{*}}{|z-w|},
$$

where $a^{*}=a / c$. Theorem 1 implies that $f$ belongs to $\operatorname{Lip}_{\alpha}(D)$.

In view of (7), Theorem 3 has as an immediate consequence:

Corollary 2. Let $f$ be a conformal mapping of a domain $D$ with property (9) onto $B$. Then $f$ belongs to $\operatorname{Lip}_{\alpha}(D)$ for $\alpha=1 / 2$.

It is not difficult to exhibit domains $D$ for which (9) and (10) are valid, with $\alpha>1 / 2$, but which are not Jordan domains - an infinite strip, for example - or which even have non-accessible boundary points. Domains satisfying (9) include a class of domains studied in [11], the domains which are $b$-arcwise connected for some $b \geqq 1$. A simply connected proper subdomain $D$ of $C$ is said to be $b$-arcwise connected if each pair of points $z$ and $w$ of $D$ can be joined in $D$ by an arc $\gamma^{*}$ satisfying dia $\left(\gamma^{*}\right) \leqq b|z-w|$. If $D$ is $b$-arcwise connected, then each pair of points $z$ and $w$ on $\partial D$ are the terminal points of a cross-cut $\gamma^{*}$ of $D$ for which dia $\left(\gamma^{*}\right)<2 b|z-w|$. A theorem of Gehring and Hayman [3] implies that the unique geodesic cross-cut 
$\gamma$ of $D$ terminating in $z$ and $w$ satisfies

$$
\max _{\zeta \in \gamma} d(\zeta, \partial D) \leqq \operatorname{dia}(\gamma) \leqq k \operatorname{dia}\left(\gamma^{*}\right) \leqq 2 k b|z-w|,
$$

where $k$ is an absolute constant. Therefore (9) holds with $c=2 k b$. Consequently, Theorem 3 yields:

Corollary 3. Let $f$ be a conformal mapping of a b-arcwise connected domain $D$ onto $B$ and let $1 / 2<\alpha \leqq 1$. Then $f$ belongs to $\operatorname{Lip}_{\alpha}(D)$ if and only if condition (10) holds.

One special case of Corollary 3 merits singling out. Among the domains which are $b$-arcwise connected for some $b$ is the class of quasidisks [5]. A domain $D$ in $C$ is a quasidisk if $D$ is the image of $B$ under a quasiconformal self-mapping of the extended complex plane. We have:

Corollary 4. Let $f$ be a conformal mapping of a quasidisk $D$ onto $B$ and let $1 / 2<\alpha \leqq 1$. Then $f$ belongs to $\operatorname{Lip}_{\alpha}(D)$ if and only if condition (10) holds.

We conclude this section by illustrating how Corollary 3 can be used to extract information on the Hölder continuity of a conformal mapping from concrete euclidean geometric data. We consider a domain along the boundary of which it is possible to "roll" a disk of fixed radius.

Corollary 5. Let $D$ be a bounded Jordan domain. Suppose there exists an $r>0$ for which the following is true: corresponding to each point $z$ on $\partial D$, there exists a closed disk of radius $r$ in $C \backslash D$ containing $z$. Then any conformal mapping of $D$ onto $B$ belongs to $\operatorname{Lip}_{\alpha}(D)$ for $\alpha=1$.

Proof. We first observe that the "disk rolling condition" has the following essentially obvious consequence for the exterior $D^{*}$ of $D$ in the extended complex plane $\hat{C}$ : there exists a constant $c>0$ such that each point $w$ of $\partial D$ is the terminal point of an end-cut $E$ of $D^{*}$ from $\infty$ satisfying

$$
\operatorname{dia}\left(E_{z}\right) \leqq c d(z, \partial D)
$$

for all points $z$ on $E$, where $E_{z}$ denotes the subarc of $E$ with $z$ and $w$ as its endpoints. Theorem 1 in [12] then guarantees the existence of a constant $M>0$ such that

$$
\operatorname{dia}(C(z, w)) \leqq M \operatorname{dia}\left(\gamma^{*}\right),
$$

whenever $z$ and $w$ are distinct points of $\partial D$ and $\gamma^{*}$ is a cross-cut of $D^{*}$ terminating in $z$ and $w$. Here $C(z, w)$ indicates the arc of smaller diameter on $\partial D$ with endpoints $z$ and $w$. (The result in [12] just cited is actually stated only for a bounded domain. The situation at hand is easily reduced to that case, however, by means of an inversion.) Nexit, an elementary argument involving (11) shows that any pair of points 
$z$ and $w$ in $D$ can be joined by an $\operatorname{arc} \gamma$ in $\bar{D}$ with

$$
\operatorname{dia}(\gamma) \leqq M|z-w| \text {. }
$$

This, in turn, insures that $D$ is $b$-arcwise connected for any $b$ larger than $M$.

In order to apply Corollary 3, we must verify (10) with $\alpha=1$. Fix a point $z_{0}$ in $D$. We may assume that $r \leqq d\left(z_{0}, \partial D\right)$. Let $z$ be any point of $D$. We show that

with $a=2 r / 3$.

$$
h_{D}\left(z_{0}, z\right) \geqq \log \frac{a}{d(z, \partial D)},
$$

Select a point $\zeta$ on $\partial D$ with the property that $d(z, \partial D)=|\zeta-z|$ and a closed disk $K$ of radius $r$ in $C \backslash D$ which contains $\zeta$. Set $D_{0}=\hat{C} \backslash K$. We have heretofore confined our discussion of the hyperbolic metric to finite domains, but the definition carries over to any simply connected subdomain of $\hat{\boldsymbol{C}}$ with nondegenerate complement. In particular, $D_{0}$ has a hyperbolic metric. If $w_{0}$ denotes the center of $K$, it is easily verified that

$$
h_{D_{0}}(\infty, w)=\log \frac{\left|w-w_{0}\right|+r}{\left|w-w_{0}\right|-r}
$$

for any $w$ in $D_{0}$. Owing to the choice of $\zeta$, it follows without difficulty that

$$
h_{D_{0}}(\infty, z)=\log \frac{2 r+d(z, \partial D)}{d(z, \partial D)}>\log \frac{2 r}{d(z, \partial D)} .
$$

Furthermore, because $r \leqq d\left(z_{0}, \partial D\right)$, it is clear that $\left|z_{0}-w_{0}\right| \geqq 2 r$, which leads to the estimate

$$
h_{D_{0}}\left(\infty, z_{0}\right) \leqq \log 3 .
$$

Finally, the fact that $D_{0}$ contains $D$ permits us to conclude from an elementary property of hyperbolic metrics that

$$
h_{D}\left(z_{0}, z\right) \geqq h_{D_{0}}\left(z_{0}, z\right) \geqq h_{D_{0}}(\infty, z)-h_{D_{0}}\left(\infty, z_{0}\right) \geqq \log \frac{a}{d(z, \partial D)}
$$

for $a=2 r / 3$, as asserted.

\section{Quasi-isometries}

A complex-valued function $f$ on a set $A$ in $C$ is called a quasi-isometry if there is a constant $L>0$ such that

$$
\frac{|z-w|}{L} \leqq|f(z)-f(w)| \leqq L|z-w|
$$

for all $z$ and $w$ in $A$. We conclude this paper by recording several characterizations of the plane domains $D$ with the property that conformal mappings between $D$ and 
$B$ are quasi-isometries. The first such characterization is merely a special case of Corollary 1.

Theorem 4. Let $f$ be a conformal mapping of a domain $D$ onto $B$. Then $f$ is a quasi-isometry if and only if, corresponding to some (each) point $z_{0}$ in $D$ there exist constants $a>0$ and $b>0$ such that

$$
\log \frac{a}{|z-w|} \leqq h_{D}\left(z_{0}, \gamma\right) \leqq \log \frac{b}{|z-w|},
$$

whenever $z$ and $w$ are distinct points on $\partial D$ and $\gamma$ is a geodesic cross-cut of $D$ terminating in $z$ and $w$.

Since the quasi-isometric image of $B$ obviously satisfies condition (9) in Theorem 3, we can combine Theorem 3 with the Becker-Pommerenke result quoted in the introduction to obtain:

Theorem 5. Let $f$ be a conformal mapping of a domain $D$ onto $B$. Then $f$ is a quasi-isometry if and only if $D$ satisfies the following two conditions.

(i) There exists a constant $c>0$ such that

$$
\max _{\zeta \in \gamma} d(\zeta, \partial D) \leqq c|z-w|,
$$

whenever $z$ and $w$ are distinct points of $\partial D$ and $\gamma$ is a geodesic cross-cut of $D$ terminating in $z$ and $w$.

(ii) Corresponding to some (each) point $z_{0}$ in $D$ there exist constants $a>0$ and $b>0$ such that

for all $z$ in $D$.

$$
\log \frac{a}{d(z, \partial D)} \leqq h_{D}\left(z_{0}, z\right) \leqq \log \frac{b}{d(z, \partial D)}
$$

Example 1 shows that condition (ii) is not by itself sufficient to insure that $f$ is a quasi-isometry. In fact Theorem 3 in [7] implies the existence of a Jordan domain $\mathrm{D}$ for which (ii) holds, but for which $f$ is not a quasi-isometry. Finally, since the quasi-isometric image of $B$ is always a quasidisk, Theorem 3 yields:

Theorem 6. Let $f$ be a conformal mapping of a domain $D$ onto $B$. Then $f$ is a quasi-isometry if and only if $D$ is a quasidisk satisfying condition (ii) in Theorem 5.

\section{References}

[1] Becker, J., and Сн. Pommerenke: Hölder continuity of conformal mappings and non-quasiconformal Jordan curves. - Comment. Math. Helv. 57, 1982, 221-225.

[2] Collingwcod, E. F., and A. J. Lohwater: The theory of cluster sets. - Cambridge Univ. Press, Cambridge, 1966.

[3] Gehring, F. W., and W. K. Hayman: An inequality in the theory of conformal mapping. J. Math. Pures Appl. (9) 41, 1962, 353-361. 
[4] Gehring, F. W., W. K. Hayman, and A. Hinkkanen: Analytic functions satisfying Hölder conditions on the boundary. - J. Approx. Theory 35, 1982, 243-249.

[5] Gehring, F. W., and B. G. OsGood: Uniform domains and the quasi-hyperbolic metric. J. Analyse Math. 36, 1979, 50-74.

[6] Gehring, F. W., and B. PALKA: Quasiconformally homogeneous domains. - J. Analyse Math. 30, 1976, 172-199.

[7] LeSLey, F. D.: On interior and exterior conformal mappings of the disk. - J. London Math. Soc. (2) 20, 1979, 67-78.

[8] LeSLEY, F. D.: Hölder continuity of conformal mappings at the boundary via the strip method. - Indiana Univ. Math. J. 31, 1982, 341-354.

[9] LesLey, F. D.: Conformal mappings of domains satisfying a wedge condition. - Preprint.

[10] NÄKKI, R., and B. PALKA: Quasiconformal circles and Lipschitz classes. - Comment. Math. Helv. 55, 1980, 485-498.

[11] NÄKKI, R., and B. PALKA: Lipschitz conditions, $b$-arcwise connectedness and conformal mappings. - J. Analyse Math. 42, 1982/83, 38-50.

[12] Pommerenke, Ch.: One-sided smoothness conditions and conformal mapping. - J. London Math. Soc. (2) 26, 1982, 77-88.

[13] Tamrazov, P. M.: Contour and solid structural properties of holomorphic functions of a complex variable. - Uspehi Mat. Nauk 28:1, 1973, 131-161 (Russian).

[14] Warschawski, S. E.: On Hölder continuity at the boundary in conformal maps. - J. Math. Mech. 18, 1968/69, 423-427.

[15] WolfF, J.: Sur la représentation conforme des bandes. - Compositio Math. 1, 1934, 217-222.

University of Jyväskylä

Department of Mathematics

SF-40100 Jyväskylä

Finland

Received 12 March 1984
The University of Texas

Department of Mathematics

Austin, Texas 78712

USA 